\title{
DAMAGE IDENTIFICATION METHOD WITH STRUCTURAL FREQUENCY DATA FROM MODAL TEST
}

Testing and analysis technology based on vibration mode is a common method to structural health monitoring and checking. This paper deduces the damage factor used for structural damage evaluation based on test frequency of structure, makes a finite element numerical simulation using ANSYS software and model test to a pressure pipe. Here a stiffness reduction method is utilized to reproduce the damage state of a single-point and multiple-point, damage identification is carried out with a FEM model of redundant pipe. Meanwhile, a model experiment system is designed to validate the efficiency of damage factor with cutting-groove to simulate damage states. Numerical and experimental results show the proposed damage factor is theoretically breviate, simple realization and high-accuracy performance, therefore, it has a good usage prospect in the implementation of structural damage monitoring and identification.

Key words: Vibration mode; Structural damage evaluation, Damage factor, Damage state

\section{Introduction}

Technology of structural experiment modal analysis is an important measure for structural damage evaluation, which is based on a modal analysis, predicts and evaluates a variation of physical parameter according to the variation of a modal characteristic of pre-damage and post-damage of the structure [1]. The development and application of technology of structural experiment modal analysis is restricted because the modal data beyond all freedoms can not be measured in view of incompleteness of test data on spot. The measure of structural frequency is much easier and precise relative to the collected data of a vibration mode, and high frequency can be measured [2]. This paper deduces the evaluation method based on the variation ratio of squared frequency and the validity is checked from a numerical simulation and model experiment test validation. Numerical and experimental results show the proposed damage factor is theoretically breviate, simple realization and high-accuracy performance; therefore, it has a good usage prospect in the implementation of structural damage monitoring and identification.

The variation of structural physical parameters must result in the variation of structural modal parameters. Damage at a specialized position has different effect on each modal characteristic, and the difference is just the basis to detect the damage position. The relationship of damage and variation in a modal parameter can be found with the perturbation theory and structure equation of motion on the basis of structural damage of a certain degree $[3,4]$.

For a multiple-degree structural system, ignoring the effect of damp, vibration eigenvalue equation is

$$
(K-\lambda \cdot M) \cdot \varphi=0
$$

where $M$ is the system mass matrix, $K$ is the system stiffness matrix, $\lambda$ is the eigenvalue and $\varphi$ is the normalized vibration mode.

While the parameters of stiffness and mass of the structure have a little variation of $\Delta K$ and $\Delta M$, from perturbation theory Eq. (1), we obtain

$$
[(K+\Delta K)-(\lambda+\Delta \lambda) \cdot(M+\Delta M)] \cdot(\varphi+\Delta \varphi)=0(2)
$$

Generally, structural damage was induced by crack or corrosion and has little effect on mass distribution of an integral structure, that is to say $\Delta M \approx 0$. On expanding Eq. (2) and ignoring the effect of $\Delta M \cdot \Delta \varphi$ and $\Delta \lambda \cdot M \cdot \Delta \varphi$, we obtain

$$
\Delta \lambda=\frac{\varphi^{T} \cdot \Delta K \cdot \varphi}{\varphi^{T} \cdot M \cdot \varphi}
$$
follows

For the $i$-th mode of vibration, Eq. (3) may be written as

$$
\Delta \lambda_{i}=\frac{\varphi_{i}^{T} \cdot \Delta K \cdot \varphi_{i}}{\varphi_{i}^{T} \cdot M \cdot \varphi_{i}} \quad(i=1,2, \ldots n)
$$

Using $\Delta K_{n}$ to donate the stiffness variation in the $n$th element, the Eq. (4) changes into

$$
\Delta \lambda_{i}=\frac{\sum_{n} \varphi_{i}^{T} \cdot \Delta K_{n} \cdot \varphi_{i}}{\varphi_{i}^{T} \cdot M \cdot \varphi_{i}}
$$

Eq. (5) is similar with Rayleigh Quotient in shape, which indicates the relationship between strain energy and structure eigenvalue [5]. Only when damage occurred on the $n$th element

\footnotetext{
* Du Jian-hua ${ }^{1}$, Ouyang Zhiwei ${ }^{2}$, Zhao Yangdong ${ }^{3}$

${ }^{1}$ School of Transportation, Shijiazhuang Institute of Railway Technology, Shijiazhuang, China, E-mail: sirtdjh@163.com

${ }^{2}$ School of Civil Engineering, Harbin Institute of Technology, Harbin, China, E-mail: 5903098@163.com

${ }^{3}$ First Branch, CCCC First Highway Consultants Co., Ltd, Xi'an, China
} 


$$
\Delta \lambda_{i}=\frac{\varphi_{i}^{T} \cdot \Delta K_{n} \cdot \varphi_{i}}{\varphi_{i}^{T} \cdot M \cdot \varphi_{i}}
$$

The eigenvalue of variation, $\lambda$ can be defined as the function of damage location $n$ and damage degree $\partial_{n}$

$$
\Delta \lambda_{i}=\lambda_{i}\left(\partial_{n}, n\right)
$$

Then $\Delta K_{n}=\partial_{n} \cdot K_{n}$, and Eq. (6) can be reduced to the following expression

$$
\Delta \lambda_{i}=\frac{\partial_{n} \varphi_{i}^{T} \cdot K_{n} \cdot \varphi_{i}}{\varphi_{i}^{T} \cdot M \cdot \varphi_{i}}
$$

From Eq. (8) we know that the variation in eigenvalue is the function damage position and damage degree. Assuming the twoorder variation eigenvalue $\Delta \lambda_{i}$ and $\Delta \lambda_{j}$ which correspond to two modes and the corresponding frequency variation ratio is

$$
\frac{\Delta \lambda_{i}}{\Delta \lambda_{j}}=\frac{\frac{\varphi_{i}^{T} \cdot K_{n} \cdot \varphi_{i}}{\varphi_{i}^{T} \cdot M \cdot \varphi_{i}}}{\frac{\varphi_{j}^{T} \cdot K_{n} \cdot \varphi_{j}}{\varphi_{j}^{T} \cdot M \cdot \varphi_{j}}}
$$

Eq. (9) shows that the variation ratio of squared frequency of any two modes is only the function of damage position, and damage of element in different position is corresponding to a set of variation ratio of squared frequency. The damage position can be identified and damage degree can be evaluated according to the variation ratio of squared frequency of each mode.

\section{Numerical simulation}

A pressure pipe with one end fixed and the other end simply supported is shown in Fig. 1 and Fig. 2 gives its corresponding FFM model.

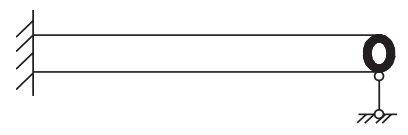

Fig. 1 Schematic of the structural model
분 12

Fig. 2 Meshing of the structure

For the purpose of making a dynamic analysis to the structure using FEM software ANSYS, the finite element model of the structure consists of 12 elements and 13 nodes. The values for the geometric properties are assigned as follows: (1) the length of the pipe $l=2400 \mathrm{~mm}$; (2) the outer diameter $D=70 \mathrm{~mm}$; and (3) the thickness of the pipe $t=25 \mathrm{~mm}$. Values for the material properties of the beam elements are assigned as follows: (1) the elastic modulus $E=72 \mathrm{GPa}$; (2) Poisson's ratio $v=0.3$; and (3) the linear mass density $\rho=2800 \mathrm{~kg} / \mathrm{m}^{3}$. Using stiffness reduction to simulate the damage of the structure member, the nine kinds of cases of pre-damage and post-damage are: - undamaged ${ }^{\sim}$ - damage at a single-point (the stiffness of element three, six, eight and eleven decreases by $10 \%$ respectively); - damage degree increases in turn (the stiffness of element five decreases by $10 \%, 15 \%$ and $20 \%$ respectively); - damage at a multiple-point (the stiffness of element three and eleven decreases by $10 \%$ at the same time), the results are shown as Table 1.

Making a comparison of cases of pre-damage and post-damage, letting the variation ratio of squared frequency be $\eta=\Delta \lambda_{j} / \Delta \lambda_{i}$ (the variation ratio of first five mode and the first mode), using the vibration number $n$ as the horizontal coordinates and $\eta$ as the vertical coordinates, the results are shown in Fig. 3.

From the figures we know that damage in different positions has different effect on the variation ratio of squared frequency of the structure and relates with the connection conditions of the structure with other structure. With the damage position away from the fixed end, the value of the variation ratio of squared frequency $\eta$ decreases gradually and then increases again at the simply supported end. Damage at a multiple-point contains the information of damage at a single-point and the variation rule is similar with the above conditions. When the damage degree increases, $\eta$ also gradually decreases. It shows that the different damage degree has a multiple relation with the damage index $\partial_{n}$. Damage can be

\begin{tabular}{|c|c|c|c|c|c|c|c|c|c|}
\hline \multirow{3}{*}{ Mode } & \multicolumn{9}{|c|}{ Damage cases } \\
\hline & \multirow{2}{*}{ Undamaged } & \multicolumn{4}{|c|}{$\begin{array}{l}\text { Damage at single-point } \\
\text { Element/degree }\end{array}$} & \multicolumn{3}{|c|}{$\begin{array}{c}\text { Damage degree increases gradually } \\
\text { Element/degree }\end{array}$} & \multirow{2}{*}{$\begin{array}{c}\text { Damage at multiple-point } \\
\text { Element/degree }\end{array}$} \\
\hline & & $3 / 10 \%$ & $6 / 10 \%$ & $8 / 10 \%$ & $11 / 10 \%$ & $5 / 10 \%$ & $5 / 15 \%$ & $5 / 20 \%$ & \\
\hline 1 & 39.306 & 39.272 & 39.113 & 38.993 & 39.228 & 39.223 & 39.176 & 39.123 & 39.194 \\
\hline 2 & 127.27 & 127.00 & 126.84 & 126.99 & 126.58 & 126.35 & 125.83 & 125.26 & 126.31 \\
\hline 3 & 265.22 & 263.45 & 264.08 & 264.22 & 263.14 & 265.07 & 264.98 & 264.88 & 261.39 \\
\hline 4 & 452.94 & 450.19 & 450.37 & 449.58 & 449.33 & 449.96 & 448.30 & 446.49 & 446.59 \\
\hline 5 & 528.60 & 524.26 & 525.85 & 527.09 & 528.41 & 525.24 & 523.30 & 521.13 & 524.08 \\
\hline
\end{tabular}
located and damage degree can be evaluated preliminarily using

The frequency $(\mathrm{Hz})$ of FEM modal analysis

Table 1 


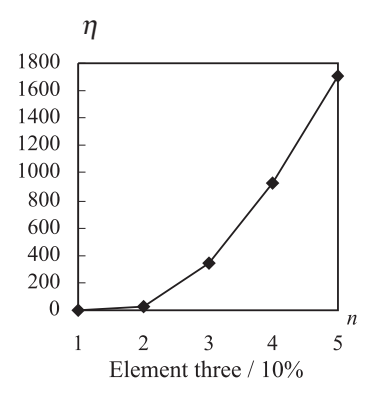

a

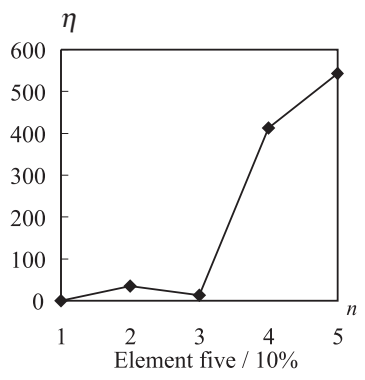

e
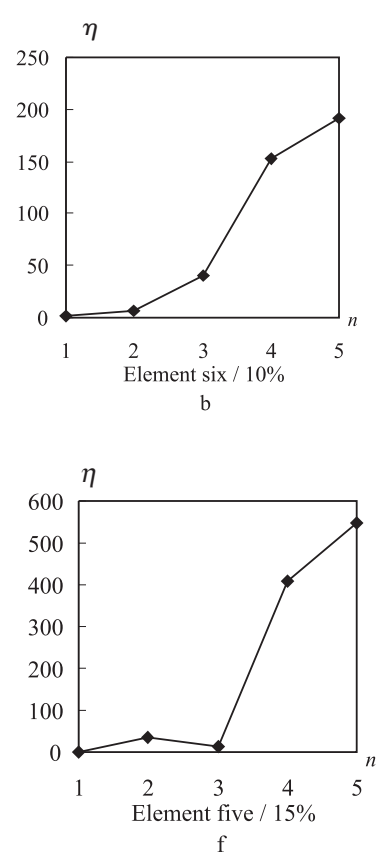
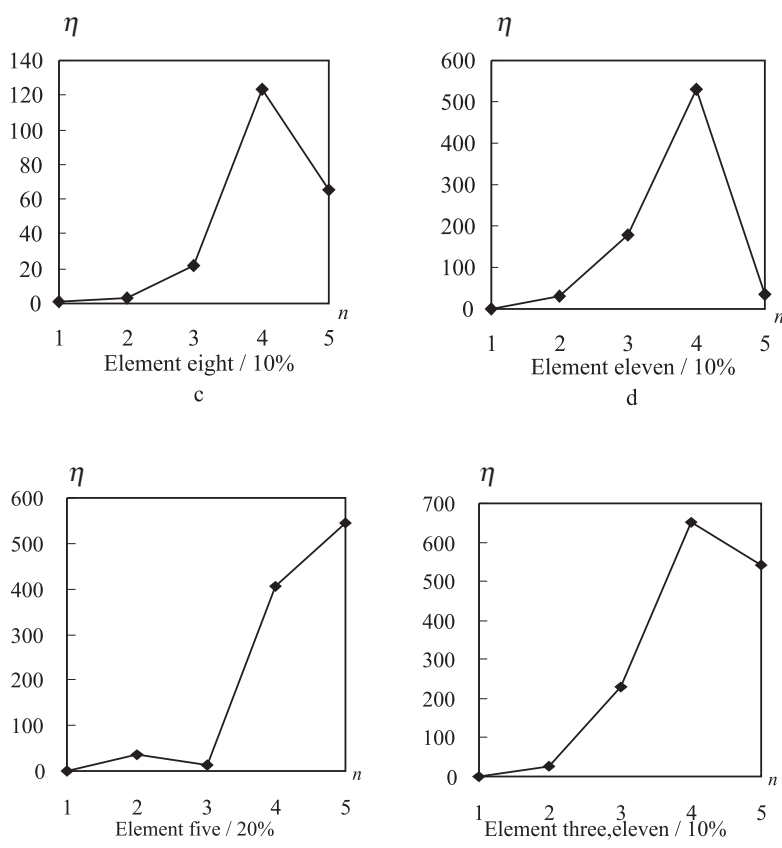

$\mathrm{g}$

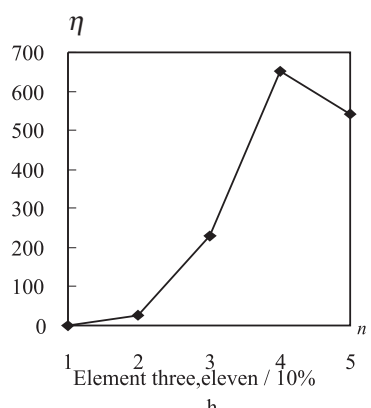

Fig. 3 Variation ratio of squared frequency

the variation ratio of squared frequency because the information of the whole structure can be expressed by the information of the elements [6].

\section{Model test}

\subsection{Test system}

The test system and model are shown in Fig. 4.

The modal test is made using a pressure pipe model. The beam is divided into twelve elements from the fixed end evenly.

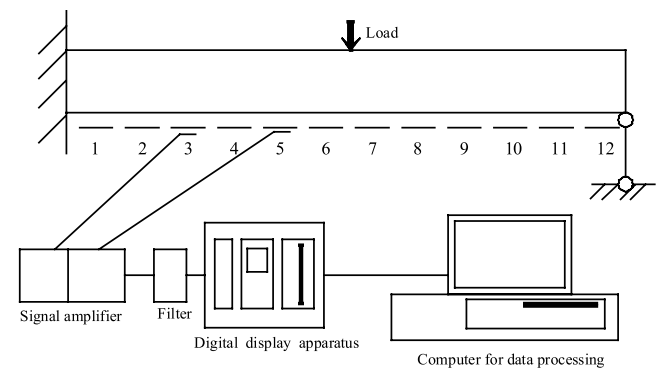

Fig. 4 Schematic of test system

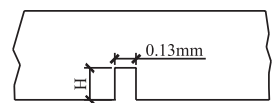

Fig. 5 Damage state
A foil electric resistance strain gage of $120 \pm 0.5 \Omega$ is pasted at the middle location of each element along the axis on the beam.

The structure makes free vibration at the pulsed excitation. After the strain response the signal is picked by a strain sensor, the strain response signal is amplified via YD-15 dynamic stain gauge, by entered dynamic signal digital display apparatus NICOLERT-2090, amplified again and filtered, at last turned into a digital signal, input into the computer and processed with ITDM (Ibriham Time Domain Method) program. The modal parameters such as natural frequency, strain modal characteristic and damp ratio can be identified.

\subsection{Damage simulation}

First, at the undamaged state of the beam structure, the vibration modal characteristic of the structure can be obtained using a single point impulse. The modal characteristic analysis is made after an impulse and response signal entered the dynamic stain gauge. The first five modal characteristics are obtained through the test, and the coherent function is quite good. The damage state on a beam section is simulated by cutting a groove which has a width of $0.13 \mathrm{~mm}$, varies with depth and is eroded by a thread with a diameter of $0.1 \mathrm{~mm}$, and the vibration test is performed.

Three cases of vibration test are performed: - undamaged; damage at a single-point (damage occurs at element three, eleven; damage occurs at element five using the increasing thickness of 2 $\mathrm{mm}, 4 \mathrm{~mm}$ and $6 \mathrm{~mm}$ in turn to simulate the damage state of the structure); - damage occurs at a multiple-point (damage occurs at 
element three and element eleven using the thickness of $2 \mathrm{~mm}$ to simulate the damage).

\subsection{Data processing and result analysis}

Table 2 is the experiment test data. After making an analysis of the test results, the relationship drawing can be worked out as shown in Fig. 6 a $f$.

From the results of data processing we know that the structural frequency which is related with the characteristic of the structure such as restraint conditions except for the damage position and damage degree shows a definite variety rule under damage at a single-point and multiple-point.

\section{Conclusion}

(1) There has been the function relationship between the variation ratio of squared frequency of pre-damage and cases of damage at multiple-point and between the variation ration of squared frequency of pre-damage and different damage degree.

(2) The analysis results show that the damage evaluation can be performed if only the former two rank variation ratio of squared frequency is acquired, but the method can not make damage state identification for symmetrical elements of the structure. The shortage can be made up with the vector data, while the damage of actual structure will not be symmetrical absolutely.

(3) The damage of a great number of structures can be denoted by using scalar quantity $\Delta K_{n}=\partial_{n} \cdot K_{n}$ to express the reduction of

The frequency $(\mathrm{Hz})$ of model test

Table 2

\begin{tabular}{|c|c|c|c|c|c|c|c|}
\hline \multirow{3}{*}{ Mode } & \multicolumn{7}{|c|}{ Damage cases } \\
\cline { 2 - 7 } & \multirow{2}{*}{} & \multirow{2}{*}{$\begin{array}{c}\text { Undamaged } \\
\end{array}$} & \multicolumn{2}{|c|}{$\begin{array}{c}\text { Damage at single-point } \\
\text { Element/H }\end{array}$} & \multicolumn{2}{|c|}{$\begin{array}{c}\text { Damage degree increases gradually } \\
\text { Element/H }\end{array}$} & $\begin{array}{c}\text { Damage at multiple-point } \\
\text { Element/H }\end{array}$ \\
\cline { 2 - 8 } & $3 / 2 \mathrm{~mm}$ & $11 / 2 \mathrm{~mm}$ & $5 / 2 \mathrm{~mm}$ & $5 / 4 \mathrm{~mm}$ & $5 / 6 \mathrm{~mm}$ & $3,11 / 2 \mathrm{~mm}$ \\
\hline 1 & 40.81 & 40.75 & 40.74 & 40.73 & 40.68 & 40.64 & 40.71 \\
\hline 2 & 132.12 & 131.83 & 131.43 & 131.17 & 130.66 & 129.87 & 131.08 \\
\hline 3 & 275.28 & 273.49 & 273.16 & 275.12 & 275.01 & 274.87 & 271.23 \\
\hline 5 & 470.18 & 467.22 & 466.46 & 467.11 & 465.31 & 463.86 & 463.46 \\
\hline
\end{tabular}

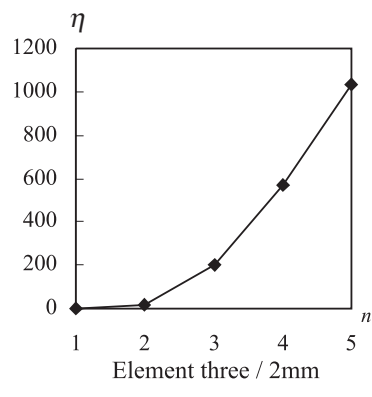

a
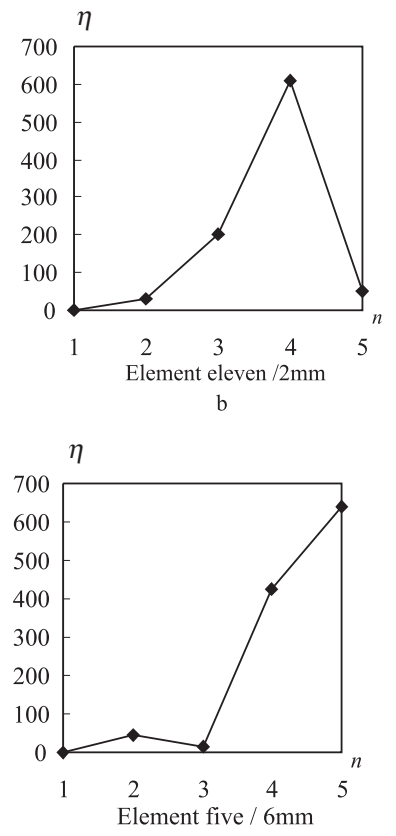

$\mathrm{e}$
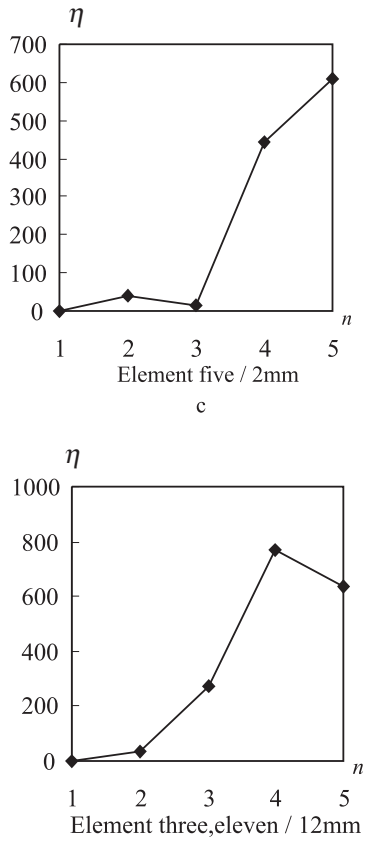

$\mathrm{f}$

Fig. 6 Variation ratio of squared frequency of damage at single-point and multiple-point of the test 
the element stiffness in the field of civil engineering, so does this paper in which damage state of the structure is simulated by the method of stiffness reduction. When applied in actual engineering, due to a high level of noise in a modal test, the measured frequency and vibration modes actually show much difference from the actual condition, and the effect of identification is affected [7]. The damage identification algorithm and checking measure which can eliminate the effect of noise should be studied further.

\section{Acknowledgement}

Here we wish to acknowledge the National High Technology Research and Development Program of China under granted number 2001AA602023-1 and my workmates with latent capacity in the Institute of Traffic Engineering, HIT, for their fund sustentation, warm support, precise comments and detailed helps, which lead to the belief that their dedication is certainly a contributing factor to this research work and it would not have been feasible without their support.

\section{References}

[1] MA, H. W., YANG, G. T.: Methods and Advances of Structural Damage Detection, Advances in mechanics, 29 (4): 513-527, 1999

[2] LI, G. Q., LOU, G. B.: The Identification of Stiffness Parameter of Frame Structure Using Frequency of Local Vibration, Journal of Vibration, measurement \& Diagnosis, 21(3): 196-201, 2001.

[3] HEARNG, TESTARB: Modal Analysis for Damage Detection in Structures, Journal of Structural Engineering, 117(11): 3042-3063, 1991.

[4] WANG, Y. G., PEI, Y. L., ZHAO, Y. D.: Vibration-Based Damage Detection with Structural Modal Characteristics, The Baltic Journal of Road and Bridge Engineering, 3 (1): 21-28, 2008.

[5] SONG, Y., XIANG, Y. Q., XU, X.: Damage Identification of Bridges Based on Vibration of Structure, Journal of Vibration, measurement \& Diagnosis, 25(3): 222-226, 2005.

[6] BYUNG, O., HWAN, H., JUNG, B. S.: Structural Assessment with Combined Data of Static and Modal Tests, Journal of Structural Engineering, 124 (8): 956-965, 1998.

[7] CAWLEY, P., ADAMS, R. D.: The Location of Defects in Structures from Measurements of the Natural Frequencies, Journal of Strain Analysis, 14(2), 49-57, 1979. 